$81 \mid 2012$

Pouvoirs du mythe dans les littératures francophones du Maghreb et du Machrek

\title{
Mythes et écriture poétique : l'exemple de Mohammed Dib
}

Fadila Chaabane

\section{OpenEdition}

\section{Journals}

Édition électronique

URL : http://journals.openedition.org/recherchestravaux/541

DOI : 10.4000/recherchestravaux.541

ISSN : 1969-6434

Éditeur

UGA Éditions/Université Grenoble Alpes

Édition imprimée

Date de publication : 30 décembre 2012

Pagination : 41-52

ISBN : 978-2-84310-238-7

ISSN : 0151-1874

\section{Référence électronique}

Fadila Chaabane, « Mythes et écriture poétique : l'exemple de Mohammed Dib », Recherches \& Travaux

[En ligne], 81 | 2012, mis en ligne le 30 juin 2014, consulté le 10 décembre 2020. URL : http://

journals.openedition.org/recherchestravaux/541 ; DOI : https://doi.org/10.4000/recherchestravaux. 541 
Fadila ChaAbane

Université d'Alger I

\section{Mythes et écriture poétique: l'exemple de Mohammed Dib}

«Le mythe, langage préexistant au texte, mais diffus dans le texte, est l'un de ces textes qui fonctionnent en lui.»

Pierre BRUNEL ${ }^{\mathrm{I}}$

La mythologie grecque, en particulier, a toujours constitué un fonds dans lequel ont puisé romanciers, poètes et dramaturges. Nombreux sont les mythes qui ont retenu l'attention des créateurs et nourri leur imaginaire : il en est ainsi des mythes d'CEdipe, du Phénix, d'Orphée, d'Antée, de Tantale, de Dédale, du Minotaure, de Vénus, d'Éros et de Thanatos pour ne citer que les plus récurrents. Nommément désigné ou implicitement référé, le mythe est omniprésent et constitue un élément inducteur important, une référence, mieux une clef de lecture du texte littéraire et du poème en particulier. Ainsi, constamment sollicités, les mythes ne cessent de dialoguer, d'interroger l'histoire et de revivre, nouveaux messagers. Dans l'écriture de la modernité, ils sont considérés comme des signes dont les poètes explorent le pouvoir d'irradiation et la richesse des connotations.

\section{Lire Éros dans Omneros}

Un parcours de quelques textes poétiques de Mohammed $\mathrm{Dib}^{2}$, poète et romancier algérien de la première génération qui n'est plus à présenter, permet de repérer, sans avoir à pousser loin l'interprétation, la présence prégnante de

I. P. Brunel, Mythocritique. Théories et parcours, PUF, I992, p. 6I.

2. Nos exemples seront puisés dans : Omneros, Seuil, 1975; Feu, beau feu, Seuil, 1979; $O$ Vive, Sindbad, Seuil, 1987 (désormais $O m, F B F$ et $O V$ dans les références après les citations). 
mythes. Le titre d'un des nombreux recueils de cet écrivain nous oriente sur cette voie : Omneros renvoie directement au mythe d'Éros, dont la présence hante l'ensemble du recueil, le plaçant sous le signe de l'hymne à l'amour, et de là, par métonymie, de l'hymne à la femme aimée. Au plan scriptural, nous pouvons relever, dans ce titre, les phonèmes constitutifs de [ $\mathrm{\varepsilon me}]$ auquel réfere métonymiquement le nom Éros (ou même directement, puisque ce mot signifie «amour charnel» en grec), ce qui inscrit donc le contenu du recueil sous un double signe mythique et noologique de l'expérience amoureuse. Dans la mythologie grecque, selon Michael Grant et John Hazel, Éros est «le dieu de l'Amour, qui aurait assuré l'union des éléments primordiaux, Ouranos (le Ciel) et Gaia (la terre)»". L'amour (Éros), selon Phèdre, comme le rapporte Platon dans Le Banquet, "est un des dieux les plus anciens, et la source des plus grands bienfaits ${ }^{4}$. " Je dirai, ajoute Agathon, que le dieu [Éros] est poète si savant qu'il rend poètes les autres à leur tour ${ }^{5}$.» La tradition orphique octroie à ce dieu «une fonction démiurgique». Il mérite bien, de ce fait, concluent ces deux poètes grecs, cités dans le même ouvrage, «tous les éloges et l'attention des hommes ${ }^{6} »$.

D'après les nombreuses occurrences des lexèmes appartement à l'isotopie d'Éros - laquelle regroupe les champs lexicaux de passion et d'amour accompagnés de toutes leurs déterminations noologiques et physiques, sans occulter le blason de l'amoureuse, en somme, tout un lexique éro(s)tique -, nous pouvons constater que l'amour et la vie occupent une place centrale dans l'œuvre analysée. Le poète l'a bien souligné en écrivant (dans le texte qui figure sur la quatrième de couverture d'Omneros) que ses textes étaient à lire «comme des poèmes d'amour et plus littéralement de l'acte d'amour même s'ils peuvent trahir la nostalgie d'une autre langue». Par ailleurs, nous noterons que chaque chapitre d'Omneros porte ce nom combiné à d'autres lexèmes qui le déterminent dans l'espace et le temps : «Éros crypte», «Éros mer», «Éros terre», «Éroslude», «Omneros", «Thanateros» et enfin «Plus noir Éros». «Un texte à lui seul peut être une répétition. Son titre redit souvent le nom d'un personnage célèbre : Médée, ou Ulysse, ou Artémis", écrit Pierre Brunel. Nous observerons, au passage, qu'intégré dans cet ensemble signifiant qu'est le titre ou/et le poème, Éros perd sa qualité de nom propre et acquiert le statut de nom commun qui a la propriété de généraliser, appartenant ainsi à

3. M. Grant, J. Hazel, Dictionnaire de la mythologie, Seghers, I975, p. I43.

4. Ibid., p. 20.

5. Platon, Le Banquet, trad. de P. Vicaire, Les Belles Lettres, 1992.

6. Ibid., p. 49.

7. P. Brunel, Mythocritique. Théories et parcours, ouvr. cité, p. 67. 
l'univers des concepts; d'où une valeur d'exemplification et d'abstraction (traits qui définissent l'archétype) dont pourrait être investi ce nom mythique dans le recueil.

Ainsi, dans la poésie de Mohammed Dib, des thèmes éternels de la littérature sont omniprésents : l'amour, la mort, la vie, combat que se livrent, sous une forme parfois épique, Éros et Thanatos, que Mohammed Dib désigne sous un mot-valise, "thanateros» $(O m, \mathrm{p}$. II2), jouant sur l'oxymore avec une volonté de conciliation toute dialectique des contraires : les pulsions de vie - que représente Éros dans la théorie freudienne - et les pulsions de mort. Éros et Thanatos sont deux noms emblématiques de la mythologie grecque, Thanatos étant la divinité de la mort, fille de $\mathrm{Nyx}$, la Nuit (elle-même engendrée par Éris). Le poète, se faisant rhapsode ou récitant, pourrait affirmer par là sa reconnaissance pour les grands poètes de l'Antiquité et définirait de la sorte l'angle générique sous lequel il faut se placer pour lire ses textes. Dans Feu, beau feu et $O$ Vive, nous avons relevé une vingtaine d'occurrences de la mort et de son champ lexico-sémantique (substantifs, verbes et adjectifs). En fait, dans les textes analysés, la mort, que désignent force métaphores et métonymies ("agonie", "croix", "râle», "deuil», "chemin de sang", $F B F$, p. 36, 80, I48 et I5O, pour ne citer que ces termes), est aussi intimement liée à la vie. Elle est sa face cachée, sa face obscure. Pour le poète, ces deux entités, naturellement antithétiques, sont parfois confondues : «le côté le plus clair de la vie, écrira Mohammed Dib dans la quatrième de couverture d'Omneros, le côté le plus perceptible est certainement le plus obscur. Il n'est que l'ombre portée d'Éros [...]». Nous soulignerons d'ailleurs, dans une perspective toute freudienne, avec Marie Bonaparte, que l'ambivalence (propre au discours poétique) est un fait primordial, élémentaire, de la vie affective humaine et qu' «un des traits des plus constants d'Éros est de traîner après soi son frère Thanatos $^{8} »$. Nous pouvons déceler là aussi, dans cette union des contraires qu'impose le mot "thanateros", une référence intertextuelle, imposant la présence implicite de l'Androgyne - thème qui occupe une place importante dans la littérature maghrébine en général et chez Abdelkébir Khatibi en particulier - «genre distinct, selon Aristophane, qui pour la forme et pour le nom tenait des deux autres, à la fois du mâle et de la femelle ${ }^{9}$.

Jouant sur les syllabes et les phonèmes de ce nom (par anagrammes), l'auteur en fait un mot de son texte, faisant proliférer les signifiants et démultipliant les signifiés : la syllabe clef («er») constitue maints lexèmes, imposant par là même, sur un autre plan de lecture, la suprématie de l'élément

8. M. Bonaparte, Chronos, Éros, Thanatos, PUF, 1952, p. 73 et I2O.

9. Platon, Le Banquet, ouvr. cité, p. 36. 
"air», indispensable à la vie et à la profération de la parole. Avec François Cheng, nous dirons bien que la poésie, comme l'est la parole mythique, est "l'état suprême et sacré de la parole ${ }^{\mathrm{ro}}$ »). Nous ajouterons que cet élément - qui évoque par métonymie l'élément "vent» et, phonétiquement, l'élément "terre» - n'est pas sans rappeler implicitement un autre mythe, Antée, (fils de Gaia, la Terre et de Poséidon, chez les Grecs, le dieu principal des mers et des cours d'eau), dont les forces se renouvelaient quand il touchait la terre. Cette dernière nourrit les rêveries : «en revenant vers la terre, le rêveur, nouvel Antée, retrouve une énergie facile, certaine, enivrante», écrit Bachelard dans L'Air et les Songes"

Pour revenir à l'univers verbal d'Omneros, nous dirons encore que ces mêmes phonèmes $[E+R]$ forment les lexèmes "ère" et "erre", mots si proches (phonétiquement et sémantiquement) de "terre» et du verbe "errer», inscription de la parole poétique dans un espace-temps non défini, à dimension mythique, celui du mythe et du conte. Les consonnes constitutives d'Omneros ne sont pas également sans évoquer ces personnages fabuleux de la mythologie grecque - qui irrigue la poésie dibienne : Hermès $([\mathrm{R}][\mathrm{m}][\mathrm{s}])$, dieu aux talons ailés, protecteur des voyageurs, Hymen ou Hyménaeos, aimé d'Apollon et personnifiant le mariage (ce nom désigne en fait le cri poussé par les invités d'une noce : o hymen, hymenaie), le célèbre Minos, fils de Zeus et d'Europe et Mnémosyne, la Mémoire, Titanide qui enfanta les Muses. À son tour, Éros convoque dans l'espace poétique dibien, par paronomase, cette autre déesse Éris, la Discorde, fille de Nyx, la Nuit qui engendra Thanatos. La nuit, qui désigne une antécédence, un passé, celui des origines, enfoui sous les limbes de la mémoire, opposée au jour et à la lumière, est aussi liée à la couleur noire (dont nous pouvons lire l'anagramme partielle dans ce titre, Omneros), couleur liée au contenu de ce recueil comme à sa thématique fondamentale : d'ailleurs, le sous-titre «Plus noir Éros» y réfere explicitement. Ces personnages mythiques que sont Éros, l'amant nocturne de Psyché, qui s'évanouit dans les airs sitôt qu'elle eut l'idée d'éclairer son visage pour savoir qui il était, un être monstrueux, comme le lui suggérèrent ses sœurs, ou un ange, et Eurydice, l'amante d'Orphée, sont aussi des êtres des ténèbres : «ils sont des fantômes de la nuit qui disparaissent, suivant leur coutume, au premier chant du coq ou au premier rayon du soleil», comme le formule Luc Benoist ${ }^{12}$.

IO. Quatre Dialogues. Rencontre entre Fr. Cheng et A. Rey, Éditions Rencontre, I969, p. 24.

II. G. Bachelard, L'Air et les Songes, José Corti, I943, p. 39.

I2. L. Benoist, Signes, symboles et mythes, PUF, coll. "Que sais-je?», I975, p. I05. 


\section{Orphée, l'obscur}

La thématique fortement présente de la nuit et de l'obscurité pourrait aussi bien évoquer cet autre être de la mythologie, Orphée, le chantre amoureux, dit le ténébreux, dont le nom justement pourrait bien dériver, selon les mythologues, d'une racine grecque du mot "obscur ${ }^{13}$ ». Le mythe d'Orphée a suscité bien des écrits, dont Les Sonnets à Orphée, de Rainer Maria Rilke. Le poète s'est identifié à ce chantre, dont la lyre ne cessa de séduire, jusqu'à son dernier souffle, «les lions, les rochers, les arbres, les oiseaux ${ }^{14}$ » (sonnet $\mathrm{n}^{\circ}{ }^{26}$ ). Mais «Si Orphée arrachant Eurydice aux enfers fournit la plus haute image des pouvoirs du chant et de l'amour, l'amant inconsolable est ensuite mis à mort par les Bacchantes qu'irrite son dédain", explique Pierre Albouy ${ }^{15}$.

Dans un de ses poèmes, Mohammed Dib écrit : «mais orphéon de chaleur/ hausse la voix importune/ et plus qu'un autre prompt/ hors de ces enceintes court/ dédier sa prière aux touffeurs» (Om, p. 55). Les termes "orphéon" et "voix" imposent la présence de ce personnage mythique. Comme l'a si bien remarqué Marcuse dans Éros et Civilisation, l'image d'Orphée - comme celle de Narcisse - est liée à la Beauté parfaite et à l'Art et se confond avec la Poésie ${ }^{16}$. «Enceintes», dans les vers cités, peut référer à un espace du dedans, l'espace de l'intimité qui enferme et étouffe, d'où "chaleur» et "touffeurs». Mais cet espace est en même temps une chapelle, un lieu spirituel où le sujet officie, «dédiant sa prière» au dieu de l'amour, sans aucun doute, et aux muses qui l'inspirent. Car, le cheminement d'Orphée pour retrouver Eurydice est symboliquement celui de l'art, c'est, écrit Maurice Blanchot dans L'Espace littéraire, «le point profondément obscur vers lequel l'art, le désir, la mort, la nuit semblent tendre ${ }^{17} »$. Ainsi, par la grâce de ces lexèmes, nous passons d'un registre à un autre, de l'érotisme et de l'exotérisme à l'ésotérisme, qui est aussi une volonté de transcendance, ce qui est la tendance propre à la poétique dibienne et, sans doute, à toute poésie.

\section{Ève, Aphrodite et le Phénix}

D'autres vers, dans Omneros, évoquent «le périple des eaux» dans une rêverie insulaire qui hante toute la poésie de Mohammed Dib :

13. P. Brunel, Mythocritique. Théories et parcours, ouvr. cité, p. 68.

I4. R. Maria Rilke, Euvres 2. Poésies [Gallimard, I926], Seuil, I972, p. 393.

I5. P. Albouy, Mythes et Mythologies dans la littérature française, Armand Colin, 1969, p. I32.

I6. H. Marcuse, Éros et Civilisation, Minuit, I968 (rééd. : I982), p. I44.

17. M. Blanchot, L'Espace littéraire, Gallimard, 1962, p. 225. 
[...] restitué simple réémergement dans le périple des eaux l'épaule le ventre les jambes paisibles puis écoulé la mer entre eux puis un pli de terre et de nouveau la solitude le rivage le bercement [...] (parabase de la p. 45).

La longueur de cette période, les séries d'énumérations, le ton solennel qui en découle, rendent bien l'aspect phénoménal de cette naissance de la femme dans un milieu aquatique, inscrite dans "un paysage aux dimensions cosmiques", dans une temporalité indéterminée (qui est celle du mythe) ou une négation de la temporalité. À partir de là, rien ne nous empêche de lire dans ce poème une référence au mythe d'Aphrodite naissant des flots. En effet, aux dires d'Hésiode, le nom de la déesse grecque de l'amour, Aphrodite, plus tard identifiée à la Vénus romaine, est dérivé de "aphos», l'écume dont elle naquit, sortant déjà femme de la mer. À Paphos, dans l'île de Chypre, elle était appelée "Anadyoméné» (celle qui sort de la mer).

Le feu, fortement occurrent dans les recueils de Mohammed Dib, lié à la parole, à la verve poétique et à la créativité, évoque aussi bien Prométhée, voleur du feu sacré, représentatif, dans l'imaginaire de nombreux écrivains, de l'acte créatif, que le Phénix; il est une métaphore de l'amour charnel et mystique. Dans la séquence : "et phénix se pétrifie» (Om, p. I28), le verbe, qui désigne une métamorphose que subit le sujet - il se transforme en pierre -, suggère une absence de mouvement, une solidification, une froideur (celle la pierre), traits sémiques qui définissent la mort, opposée à la vie qui est mouvement et chaleur. Mais cette pétrification pourrait aussi bien renvoyer à ce qui dure et vainc le temps, tel le mythe, tel le Phénix renaissant indéfiniment des flammes qui le dévorent. Le Phénix peut bien être un symbole de vie et de renaissance, "de la dialectique de la vie et de la mort», note Bachelard dans Le Droit de rêver ${ }^{18}$. Ailleurs, c'est aussi le Phénix qui est sans doute désigné par la périphrase : «l'ange brûlant» $(O V$, p. 62). Cet être mythique serait ici un élément paragrammatique, métaphorisant le poète comme créateur et amoureux, ce que peut suggérer la séquence "prescience d'eau " dans le même texte, liant aussi par là ces éléments antithétiques que sont l'eau et le feu. Dans la symbolique universelle, selon Jean Chevalier et Alain Gheerbrant, le Phénix renvoie au «feu créateur et destructeur dont le monde tient ses origines. Il rend compte aussi d'une irréfragable volonté de survie $^{19}{ }$. Ce sont donc ces dimensions du mythe que Mohammed Dib a exploitées et auxquelles réfere explicitement son œuvre.

I8. G. Bachelard, Le Droit de rêver [1970], PUF, coll. "Quadrige», 2002, p. I75.

19. J. Chevalier et A. Gheerbrant, Dictionnaire des symboles, Robert Laffont, I982, p. 748. 


\section{Ophélie et Caron}

Nous lisons ailleurs, dans le poème intitulé «publication de l'obscurité» (Om, p. I5), ces vers : «cette eau ou ces ténèbres/ ce quelque chose de commun/ qu'elles fondent sur le désastre "; le poète y associe l'élément aquatique à l'élément temporel nuit (qui est une des déterminations de la mort, avec le froid et l'immobilité), représenté métonymiquement par "ténèbres", terme qui n'est pas sans évoquer la mort et les enfers, Hadès et le Léthé. «L'imagination du malheur et de la mort trouve dans la matière de l'eau une image matérielle particulièrement puissante et naturelle», écrit Bachelard dans L'Eau et les Rêves $^{20}$. Mais une mort dans l'eau, une mort si douce ne peut-elle pas encore évoquer, pour nous lecteurs, et convoquer dans l'espace textuel un autre renvoi, cette fois-ci à un mythe littéraire, celui d'Ophélie que Bachelard a si bien analysé? L'Ophélie de Shakespeare est une créature née pour mourir dans l'eau, elle y retrouve "son propre élément ${ }^{2}{ }^{\star}$, commente le philosophe dans le même essai. Ce qui lui permet de conclure que «l'eau est l'élément de la mort jeune et belle, de la mort fleurie", renvoyant également aux œuvres d'art qui ont traité ce thème. Ce mythe d'Ophélie est aussi à lier, pour Bachelard, à l'idée de la renaissance dans les eaux.

Dans ces vers extraits de Feu, beau feu (p. 25), «[...] puis eau couvrante/ je borderai de hanches / ces conques où mourir", la séquence, "ces conques où mourir ", marque cette ambivalence fondamentale du symbolisme de la mer, à la fois source de vie et profondeurs abyssales où règne la mort. Nous préciserons que la conque pourrait également, par analogie, évoquer pour nous la barque - le mot figure dans les textes de Mohammed Dib, dans Omneros (p. 6o) et $O$ Vive (p. 6o) - dont le symbolisme est riche. Selon Gilbert Durand, la barque "concentre en elle les vertus de salvation, de fécondité, de naissance des eaux, au sein même de la tempête, de la colère aquatique et de la mort diluviale ${ }^{22} »$. Elle est liée, tout à la fois, à la mort (par le mythe littéraire de la barque de Caron), au voyage initiatique, à la rêverie, au temps et à la quête. Dans de nombreuses sépultures, rapportent les anthropologues, des miniatures de barques ont été trouvées, sans doute parce qu'elles permettent "de couper à travers les multiples états de l'être», selon Jean-Pierre Bayard ${ }^{23}$.

20. Ibid., p. I05.

2I. Ibid., p. 98.

22. G. Durand, "Le symbolisme de l'eau», dans Encyclopaedia universalis, I990, p. 892.

23. J.-P. Bayard, La Symbolique du monde souterrain, Payot, I973, p. 44. 


\section{Mort et Minotaure}

Ailleurs, dans le poème intitulé : «Lieu de femme» $(O m$, p. 3I), un autre être mythique est évoqué, le Minotaure :

où la désole un échouage / agite un chœur amer d'oiseaux / parcourent la fièvre l'aération // où l'attache une plainte frayant / la voie aux basses eaux à leur mort / selon les progrès d'une vacance // et ce qui liberté de lumière ici/ sans franchir ni passe ni mémoire / là-bas fait un bruit de minotaure.

Nous observerons que le lexème "mort», dans ces vers, est présent, anagrammatisé dans le mot "minotaure». Cet être légendaire, mi-homme, mitaureau, qui est lui aussi le symbole de la quête, «représente, selon JeanPierre Bayard, moins un monstre qu'une étape nécessaire dans l'initiation ${ }^{24}$ ». Dans la symbolique universelle, le Minotaure représente «le combat spirituel contre le refoulement qui ne peut être victorieux que grâce à des armes de lumière ${ }^{25}$ ".

Ainsi, le poète a pu rêver à ce riche symbolisme que ces mouvements des eaux marines peuvent induire. Ces eaux qui se retirent, dans un bruit de «volière de vagues» (Om, p. I26) - comme le Minotaure enfermé dans son labyrinthe -, esseulant le rivage, faisant s'échouer la voile, font penser au monde souterrain que voilent les ténèbres et qui, de ce fait, évoque directement la mort et les enfers. Le poète écrit ailleurs ( $F B F$, p. 89, 90 et I36) :

en même temps étroit cheminement [...] / réanimée par le circuit des âges requise par le chant (le champ) dis-je tu repars demande d'amnistie démantèlement de la peau et des os tu reviens tu repasses [...] et reperds la route/ [...] route comme on court/ et revient en arrière/ retrouve le regard.

Nous pouvons tout de suite remarquer la redondance des verbes de mouvement liés ici au lexème "circuit» (du latin circumire, "faire le tour") qui signale un itinéraire planifié, un trajet à parcourir pour faire le tour d'un lieu, avec l'idée d'un retour au point de départ; ce même terme peut indiquer (de par sa racine "cercle») ce qui recommence. Ainsi, l'errance dans le labyrinthe est essentiellement initiatique et le labyrinthe en lui-même peut donc être appréhendé "comme une sorte de vaste métaphore de la connaissance», dirons-nous avec André Peyronie :

[...] Il renferme le chemin conduisant à la vérité originelle [que figure] le Minotaure. [...] Fondamentalement, le mouvement du savoir est vers le Minotaure et non salut par le fil qui conduit au-dehors ${ }^{26}$.

24. Ibid., p. IO5.

25. J. Chevalier et A. Gheerbrant, Dictionnaire des symboles, ouvr. cité, p. 635.

26. A. Peyronie, "Minotaure», dans Dictionnaire des mythes littéraires, ouvr. cité, p. I057. 
Nous signalerons enfin que le mot «minotaure» figure dans le poème intitulé "Lieu de femme» $(O m$, p. 3I). Ce poème fait partie du chapitre intitulé "Éros mer", liant fortement l'amour à l'eau et à la vague en particulier; le bruit du minotaure est donc aussi, dans la continuité de cette thématique, celui de la houle qui se meut dans les profondeurs pélagiques, à l'image du monstre enfermé à l'intérieur de la terre. Nous décodons ici une autre métaphore de la bestialité du désir qu'éveille la présence de la femme, le minotaure étant l'archétype de cette perversité. André Peyronie pense que, «sous ce couvert mythologique, pourrait se traduire une sorte de culpabilité animale liée à la sexualité, en particulier à la sexualité féminine ${ }^{27}$ ».

\section{D’aloès à Éloa}

Des noms mythiques, d'autres encore sont évoqués implicitement dans un des vers de Feu, beau feu (p. 19) à partir du lexème aloès : "et l'aloès / couvre la terre/d'armes et de cris». Dans le nom latin de cette plante (Aloe vera), nous pouvons lire d'abord et doublement présent, l'anagramme d'Ève. Nous noterons que la femme aimée, dans le recueil qui porte son nom, est baptisée sous le nom de Vive (anagramme de la femme mythique, la première femme, «Ève»). Nous avons constaté également que, dans la poésie de Mohammed Dib, le motif de la nudité (lié métonymiquement à Ève), qui suscite une interprétation tant érotique qu'ésotérique, est récurrent. L'adjectif «nu»-de même que le substantif «nudité» - répété à maints niveaux des trois recueils, qui appartient au vocabulaire érotique, pourrait tout aussi bien référer au mythe adamique : dans $O$ Vive, nous signalerons ces quelques séquences : «l'immuable nue» (p. 87), "paisible nue» (p. I06), "toute nue» (p. II2), «nue» (p. 54); dans Feu, beau feu, nous lisons: "prendre ta nudité» (p. I7), «nu au paysage» (p. 25), "nudité aubier» (p. 50), "nudité encore» (p. 48), «ma nudité à nu» (p. 70). D’autres exemples pourraient être encore cités, sans compter les occurrences de la syllabe [ny], formant d'autres lexèmes anagrammatisant l'adjectif en question. La référence à la nudité pourrait être sans doute l'expression métaphorique, nous dirions même allégorique, d'une envie de remonter à la source du temps, de retrouver une antécédence, l'état d'Ève avant la chute. Ainsi, dans sa rêverie poétique, le poète conçoit une eau femme qui sait aussi bien se dévêtir : "l'eau à genoux l'eau plus nue / qui patiente aux barrières / tout aussi abandonnée» $(\mathrm{Om}, \mathrm{p} .84)$. Selon Bachelard, « eau» et «nudité» sont étroitement liées; un même trait les caractérise : leur

27. Ibid., p. 1056 . 
innocence et leur nativité. Pour un rêveur des éléments, "les êtres vraiment nus, aux lignes sans toison, sortent toujours d'un océan ${ }^{28}$ ", comme Aphrodite, dirons-nous.

Dans «aloès", le lecteur peut également identifier le nom d'Arès, amoureux d'Aphrodite et dieu de la guerre, dans la mythologie grecque, que seuls réjouissaient les batailles et les carnages. Signalons aussi ce nom des géants qui enfermèrent ce fougueux coursier dans un pot de bronze, les Aloades, anagramme quasi parfaite de "aloès»! Et personne ne pourrait nous empêcher de penser à ce nom célèbre de l'épopée romantique, celui d'Éloa, le type «du mythe de la femme rédemptrice, de la féminité bienfaisante", selon les termes mêmes de Gilbert Durand ${ }^{29}$.

\section{De la pérennité des mythes ou le poète-forgeron Protée}

Bien d'autres mythes pourraient être encore identifiés dans la trame des écrits analysés, entre autres, celui du forgeron et de sa forge, lié aux éléments, au feu, à l'air, à la terre et au fer, dont fut maître David et sur lequel nous allons nous arrêter quelque peu. Nous lisons sous la plume de Mohammed Dib, dans Omneros (pour ne citer que ces exemples) :

rien qui ne soit forge/ entre blondeur et hâle (p. 27);

et dans le plus sombre des ors / tel un lointain travail du cœur / cette lente forge à murmures (p. IO3-IO4);

femme plus que patience/ plus qu'étiage de hanches / ou forge à travers une aire (p. 129).

L'isotopie du feu, dans les recueils poétiques de Mohammed Dib, est riche en termes référant à la forgerie («forge» et «forger» sont redondants) et donc au travail de l'homme. Ainsi, les mots "forge» et «feu» renvoient directement au mythe de Prométhée, qui est celui de l'utilisation du feu par l'homme, et révèlent les progrès réalisés. Nous observerons que la rêverie de la forge est une rêverie cosmique de puissance et de domination. La forge, en littérature, manifeste la volonté du sujet d'agir pour changer le cours des choses : elle "est une des grandes rêveries de la volonté», écrit Bachelard dans La Terre et les Rêveries de la volonté ${ }^{\circ}$. La mythologie africaine fait du forgeron un héros civilisateur chargé, comme Prométhée, d'apporter aux hommes le feu dérobé

28. G. Bachelard, L'Eau et les Rêves, ouvr. cité, p. 45.

29. G. Durand, Dictionnaire des mythes littéraires, ouvr. cité, p. 264-265.

30. G. Bachelard, La Terre et les Rêveries de la volonté, José Corti, 1947 (rééd. : Cérès, Tunis, 1996), p. 166. 
aux dieux. De ce fait, la forge est perçue, dans la symbolique universelle, "comme un espace de création, le premier lieu des transformations, où le minerai devient métal puis outil. L'ouvrage humain s'y réalise à l'image de la création divine ${ }^{31}$ ». Selon Luc Benoist, «le travail du forgeron a d'abord été rituel, aussi bien céleste que chtonien", d'où sa dimension mythique. Dans la tradition biblique, ajoute-t-il, le premier ouvrier fut Tubal-Caïn. Or, en arabe, le nom de Caïn signifie "forgeron".

Par ailleurs, et à un autre niveau de lecture, la métamorphose de l'amante en louve et de l'amant en loup, dans $O$ Vive notamment, pourrait bien faire penser à Protée, le maître des métamorphoses et le dieu de la mer. Le poète écrit : "mais tu réserveras / une place au loup / dans ce velours blanc» (p. I8). Dans un des poèmes d'Omneros, intitulé «Corps à cris» (p. 59), nous lisons, pour ne citer que ce texte : «louve en un creux de désir/ toute entre ces bras recluse / qui ne cesse d'user d'ongles [...]». Plus loin, le poète utilise un néologisme : «louverie» (p. I45), désignant sans doute ainsi l'ordre constitué par ces canidés (formé probablement à partir de deux mots : «louveterie», ou chasse au loup ou/et "louveter», mettre bas en parlant de la louve). Cette forgerie vise sans doute à opérer une recatégorisation du nom «loup", animal emblématique d'Apollon, lui octroyant un statut d'archétype. Dans la tradition nordique, observe Gilbert Durand, les loups, "associés aux puissances funèbres", sont le symbole de la mort cosmique. Ils sont dévorateurs d'astres ${ }^{32}$.

Ainsi la référence à la mythologie classique et aux mythes personnels, leur intégration dans un univers langagier spécifique, orientent la lecture du texte poétique en général et du texte maghrébin d'expression française en particulier, par le fait même que le mythe est, au plan de sa fonction textuelle spécifique, un "pourvoyeur de références». Le mythe est bien, comme nous l'avons souligné plus haut, à la source même de l'écriture poétique. Les noms mythiques sont autant de signes à explorer. À l'instar d'autres écrivains, Mohammed Dib, en fait «un mot de son texte» : celui d'Éros par exemple, nous l'avons vu, est combiné aisément à d'autres noms et désigné par anagramme dans les poèmes. Sans être nommément cité, le mythe peut être textuellement présent à partir des occurrences des lexèmes qui y réfèrent par le biais des images poétiques et des figures, la métonymie en particulier : ainsi, l'enfer renvoie à l'Hadès, l'eau noire à la mort, et donc à Thanatos, le labyrinthe au Minotaure et à Orphée, le feu au Phénix, Vive nue convoque Ève et le mythe adamique,

3I. Dictionnaire encyclopédique de la langue française, Larousse, 1999, p. IIIO.

32. G. Durand, Les Structures anthropologiques de l'imaginaire, Bordas, 1969, p. 9I. 
elle est la femme aimée dont le poète joue aussi avec le nom. Mythe et métaphore - celle-ci est une figure clé du langage poétique - sont liés, plus même, le mot lui-même peut être appréhendé comme "un élément mythographique et toute métaphore peut être prise pour une courte fable», note Julia Kristeva dans Le Langage ${ }^{33}$. Dans le discours poétique, la présence de figures de style, telles que l'anaphore, la personnification et la prosopopée, l'allégorie qu'elles génèrent, «transforme aisément, dirons-nous avec Pierre Albouy, l'univers verbal en univers mythologique ${ }^{34}$ ». En fait, dans sa genèse, l'expérience poétique rejoint bien l'expérience mythique. C'est ce que rappelle Max Bilen, dans son article "Comportement mythico-poétique»:

Le récit mythique cosmogonique et le poème, écrit-il, ont une même fonction : permettre à l'homme de vivre une condition invivable [laquelle] est une manifestation de l'aspiration de l'homme vers une liberté qui sous-tend le désir de s'autocréer, c'est-à-dire de renaître selon ses vœux ${ }^{35}$.

33. J. Kristeva, Le Langage, Seuil, coll. «Points», 1981, p. 170.

34. P. Albouy, Mythes et Mythologies dans la littérature française, ouvr. cité, p. Io.

35. G. Durand, Dictionnaire des mythes littéraires, ouvr. cité, p. 358. 